\title{
Treatment outcome of radiation therapy and concurrent targeted molecular therapy in spinal metastasis from renal cell carcinoma
}

\author{
Sangjoon Park, MD, Kyung Hwan Kim, MD, Woo Joong Rhee, MD, Jeongshim Lee, MD, \\ Yeona Cho, MD, Woong Sub Koom, MD, PhD \\ Department of Radiation Oncology, Yonsei University College of Medicine, Seoul, Korea
}

\begin{abstract}
Purpose: To evaluate the clinical outcomes of patients who underwent radiation therapy with or without targeted molecular therapy for the treatment of spinal metastasis from renal cell carcinoma (RCC).

Materials and Methods: A total of 28 spinal metastatic lesions from RCC patients treated with radiotherapy between June 2009 and June 2015 were retrospectively reviewed. Thirteen lesions were treated concurrently with targeted molecular therapy (concurrent group) and 15 lesions were not (nonconcurrent group). Local control was defined as lack of radiographically evident local progression and neurological deterioration.

Results: At a median follow-up of 11 months (range, 2 to 58 months), the 1-year local progression-free rate (LPFR) was 67.0\%. The patients with concurrent targeted molecular therapy showed significantly higher LPFR than those without $(p=0.019)$. After multivariate analysis, use of concurrent targeted molecular therapy showed a tendency towards improved LPFR (hazard ratio, 0.13; $95 \%$ confidence interval, 0.01 to 1.16). There was no difference in the incidence of systemic progression between concurrent and nonconcurrent groups. No grade $\geq 2$ toxicities were observed during or after radiotherapy.

Conclusion: Our study suggests the possibility that concurrent use of targeted molecular therapy during radiotherapy may improve LPFR. Further study with a large population is required to confirm these results.
\end{abstract}

Keywords: Renal cell carcinoma, Neoplasm metastasis, Radiotherapy, Molecular targeted therapy

\section{Introduction}

Approximately $30 \%$ of the patients with renal cell carcinoma (RCC) initially present with synchronous distant metastasis, while another 30\% develop metachronous metastasis [1,2]. However, because of its radioresistant nature, the role of radiation therapy (RT) in treating primary $\mathrm{RCC}$ has been relatively limited $[3,4]$. For spinal metastasis from $R C C$, on the other hand, RT is the principle local therapy for both pain palliation and local control. Historically, spinal metastasis has been treated with external beam RT and recently, several studies have demonstrated that stereotactic body radiation therapy (SBRT) could offer durable local control and pain palliation [5-7].

Though the prognosis of metastatic RCC was dismal for many years [8], it has improved dramatically since the

Received 16 March 2016, Revised 14 April 2016, Accepted 16 May 2016.

Correspondence: Woong Sub Koom, MD, PhD, Department of Radiation Oncology, Yonsei Cancer Center, Yonsei University College of Medicine, 50 Yonsei-ro, Seodaemun-gu, Seoul 03722, Korea. Tel: +82-2-2228-8116, Fax: +82-2-312-9033, E-mail: MDGOLD@yuhs.ac

(c) This is an Open Access article distributed under the terms of the Creative Commons Attribution Non-Commercial License (http://creativecommons.org/ licenses/by-nc/4.0/) which permits unrestricted non-commercial use, distribution, and reproduction in any medium, provided the original work is properly cited.

www.e-roj.org 
introduction of targeted molecular agents such as sunitinib, a vascular endothelial growth factor (VEGF) tyrosine kinase inhibitor (TKI), and temsirolimus, a mammalian target of rapamycin (mTOR) inhibitor [9]. Antiangiogenic agents induce normalization of vasculature in the tumor, resulting in a synergistic antitumor effect when used concurrently with RT [10]. Two prospective studies have reported the feasibility of concurrent use of targeted molecular therapy and radiation in various types of cancers $[11,12]$. However, level 1 evidence supporting the benefit of concurrent use of targeted molecular therapy during the course of RT for RCC is lacking. In addition, the optimal timing of administration (before, during, or after RT) has not yet been determined.

In our institution, patients with metastatic $R C C$ receive targeted molecular therapy as the first-line systemic therapy and the metastatic lesions, spine in particular, are commonly treated with RT. The purpose of this study was to evaluate the difference in clinical outcome according to the use of concurrent targeted molecular therapy with RT in spinal metastasis from RCC.

\section{Materials and Methods}

\section{Patient selection}

From June 2009 to June 2015, 28 spinal metastatic lesions from 24 patients with pathologically confirmed RCC were treated with either SBRT or non-SBRT. A spinal metastatic lesion involving more than one contiguous spine level was counted as one treated lesion. Among patients treated, the maximum number of spine metastasis was 6 lesions. Eleven patients were excluded from this study due to incomplete RT (n $=4)$ and insufficient follow-up $(n=7)$.

Imaging studies were performed to precisely define the extent of disease before treatment. Pre-treatment computed tomography (CT) was available for all lesions. Magnetic resonance imaging (MRI) and positron emission tomography (PET) were not performed routinely and were available for 19 and 8 lesions, respectively.

\section{Radiation therapy}

Among the 28 treated lesions, 8 lesions were treated with SBRT and 20 lesions with non-SBRT. Immobilization devices were applied in patients treated with SBRT. Thermoplastic headshoulder masks were used when treating cervical lesions and customized total body vacuum bags were used for thoracic and lumbar lesions.

For SBRT, the clinical target volume was defined as the involved area of the skeletal structures. Treatment planning for SBRT was performed by helical tomotherapy using the HiArt (TomoTherapy Inc., Madison, WI, USA) planning station or by Cyberknife (Accuray Inc., Sunnyvale, CA, USA). The dose schedules were 24 Gy in 3 fractions ( $n=3), 40$ Gy in 5 fractions ( $n=1), 48$ Gy in 4 fractions $(n=1)$, and 18 Gy in single fraction $(n=3)$. The dose was prescribed at an $80 \%$ isodose level.

Non-SBRT was planned using three-dimensional RT. The involved vertebral bodies with sufficient margins and one adjacent level of spines were defined as the target volume. Various dose schedules were used, while the most frequently used schedule was $30 \mathrm{~Gy}$ in 10 fractions, ranging from 20 to $40 \mathrm{~Gy}$ in 5-12 fractions.

Biologic effective dose (BED) was calculated according to the linear quadratic model to compare the effects of various fraction sizes and total doses. We adopted an $\alpha / \beta$ value of 7 , given the radioresistant nature of RCC [13].

\section{Targeted molecular therapy}

Targeted molecular therapies were used in 22 patients, either before $(n=6)$, after $(n=5)$, or simultaneously ( $n=11)$ with RT. Two patients had not received any kind of targeted molecular therapy during the course of their disease. Either a VEGF tyrosine kinase inhibitor (sunitinib $[n=5]$, sorafenib $[n=6]$, pazopanib $[n=8]$ ) or mTOR inhibitor (temsirolimus $[n=1]$, everolimus $[n=2])$ was administered.

\section{Outcome assessment}

Follow-up CT or MRI was performed every 1-3 months after treatment for the first year and every 6 months thereafter. The response of spinal metastasis after RT was classified as controlled or progressed, according to the response assessment criteria suggested by the SPine response assessment in NeuroOncology (SPINO) group [14]. Local progression was defined as showing one of following: gross increase in tumor volume or in linear dimension, any new or progressive tumor within the epidural space, neurological deterioration attributable to pre-existing epidural disease with equivocal increased epidural disease dimensions. Progression outside of treated lesions was considered indicative of systemic progression.

A physician interviewed the patients before the start of treatment, at 1 month after RT, and every 3 months thereafter. Pain was assessed using a numerical rating scale, where 0 represented 'no pain' and 10 was 'pain as bad as you can imagine.' A decrease in pain score after treatment was considered relief of pain. 
Table 1. Patient and spine characteristics

\begin{tabular}{|c|c|c|c|}
\hline \multirow[b]{2}{*}{ Characteristic } & \multirow{2}{*}{$\begin{array}{c}\text { Total } \\
(n=24)\end{array}$} & \multicolumn{2}{|c|}{ Target therapy } \\
\hline & & $\begin{array}{l}\text { Nonconcurrent/none } \\
\qquad(\mathrm{n}=13)\end{array}$ & $\begin{array}{l}\text { Concurrent } \\
(n=11)\end{array}$ \\
\hline Age (yr) & $65(34-82)$ & - & - \\
\hline \multicolumn{4}{|l|}{ Sex } \\
\hline Male & $16(66.7)$ & $8(61.5)$ & $8(72.7)$ \\
\hline Female & 8 (33.3) & $5(38.5)$ & $3(27.3)$ \\
\hline \multicolumn{4}{|l|}{ ECOG PS } \\
\hline $0-1$ & $14(58.3)$ & 7 (53.8) & 7 (63.6) \\
\hline $2-4$ & $10(41.7)$ & $6(46.2)$ & $4(36.4)$ \\
\hline Spine & 28 & 15 & 13 \\
\hline \multicolumn{4}{|l|}{ Location of spine tumor } \\
\hline Cervical & $1(2.8)$ & - & - \\
\hline Thoracic & $9(25.0)$ & - & - \\
\hline Lumbar & $16(44.4)$ & - & - \\
\hline Sacral & $10(27.8)$ & - & - \\
\hline \multicolumn{4}{|l|}{ Cord compression } \\
\hline No & $20(71.4)$ & $10(66.7)$ & $10(76.9)$ \\
\hline Yes & $8(28.6)$ & $5(33.3)$ & $3(23.1)$ \\
\hline \multicolumn{4}{|l|}{ SINS } \\
\hline $0-6$ & $9(32.1)$ & $5(33.3)$ & $4(30.8)$ \\
\hline $7-12$ & $18(64.3)$ & $9(60.0)$ & $9(69.2)$ \\
\hline $13-18$ & $1(3.6)$ & $1(6.7)$ & $0(0)$ \\
\hline \multicolumn{4}{|l|}{ Prior irradiation } \\
\hline No & $24(85.7)$ & $13(86.7)$ & $11(84.6)$ \\
\hline Yes & $4(14.3)$ & 2 (13.3) & $2(15.4)$ \\
\hline \multicolumn{4}{|l|}{ Dose scheme } \\
\hline Non-SBRT & $20(71.4)$ & $11(73.3)$ & $9(69.2)$ \\
\hline SBRT & 8 (28.6) & $4(26.7)$ & $4(30.8)$ \\
\hline Dose (BED) & $51.4(31.4-130.3)$ & & \\
\hline$<51.4$ & $12(42.9)$ & $6(40.0)$ & $6(46.2)$ \\
\hline$\geq 51.4$ & $16(57.1)$ & $9(60.0)$ & $7(53.8)$ \\
\hline \multicolumn{4}{|l|}{ Pain response } \\
\hline No & $10(35.7)$ & $5(33.3)$ & $5(38.5)$ \\
\hline Yes & $18(64.3)$ & $10(66.7)$ & $8(61.5)$ \\
\hline
\end{tabular}

Values are presented as median (range) or number (\%).

ECOG PS, Eastern Cooperative Oncology Group performance status; SINS, Spinal Instability Neoplastic score; SBRT, stereotactic body radiation therapy; $B E D$, biological effective dose.

\section{Statistical analysis}

Local progression-free rate (LPFR) and overall survival (OS) were defined as the time from start of RT to local progression and to death from any cause, respectively. LPFR and OS were estimated using the Kaplan-Meier method and were compared using a log-rank test. To determine factors associated with LPFR after RT, Cox proportional hazards method was used at both univariate and multivariate levels. Factors proven to be significant in univariate analysis were entered in a multivariate analysis. Hazard ratios (HR) and corresponding 95\% confidence intervals $(\mathrm{Cl})$ were calculated. A p-value $<0.05$ was considered to be significant in all statistical analysis. The data were analyzed using IBM SPSS ver. 20 (IBM, Armonk, NY, USA).

\section{Results}

\section{Patient and spine characteristics}

The patient and spine characteristics for 28 lesions from 24 patients are summarized in Table 1. In 3 patients, there were multiple spine lesions treated, resulting in a total of 28 treated lesions. Twenty-two patients (91.7\%) were treated 
Table 2. Results of univariate and multivariate analyses of local progression-free rate

\begin{tabular}{|c|c|c|c|c|c|c|}
\hline \multirow{2}{*}{ Variable } & \multicolumn{3}{|c|}{ UVA } & \multicolumn{3}{|c|}{ MVA } \\
\hline & $\mathrm{HR}$ & $95 \% \mathrm{Cl}$ & p-value & $H R$ & $95 \% \mathrm{Cl}$ & $p$-value \\
\hline ECOG PS & 0.79 & $0.21-2.98$ & 0.726 & - & - & - \\
\hline Target therapy & 0.12 & $0.02-0.98$ & 0.048 & 0.13 & $0.01-1.16$ & 0.068 \\
\hline SINS group & 0.86 & $0.21-3.62$ & 0.837 & - & - & - \\
\hline Cord compression & 7.38 & $1.54-35.28$ & 0.012 & 4.28 & $0.78-23.39$ & 0.093 \\
\hline Prior irradiation & 1.10 & $0.22-5.45$ & 0.905 & - & - & - \\
\hline Dose scheme & 0.75 & $0.15-3.74$ & 0.729 & - & - & - \\
\hline Radiation dose (BED) & 0.52 & $0.13-2.11$ & 0.357 & - & - & - \\
\hline Pain response & 0.23 & $0.05-0.96$ & 0.044 & 0.19 & $0.04-0.92$ & 0.039 \\
\hline
\end{tabular}

UVA, univariate analysis; MVA, multivariate analysis; $\mathrm{HR}$, hazard ratio; $\mathrm{Cl}$, confidence interval; ECOG PS, Eastern Cooperative Oncology Group performance status; SINS, Spinal Instability Neoplastic score; BED, biological effective dose.

with targeted molecular therapy at least once during the course of their disease. Of these patients, 13 (54.2\%) had received targeted molecular therapy concurrently with $\mathrm{RT}$. Those without targeted molecular therapy or treated with target agents but not simultaneously with radiation were classified as "Not concurrent/none" group. Eight lesions (28.6\%) involved suspected spinal cord compression, but there was no definite evidence of neurologic deficit. The pre-treatment Spinal Instability Neoplastic score (SINS) was calculated for 28 spine lesions [15]. Twenty spine lesions were treated with non-SBRT with a median BED of 51.4 Gy (range, 31.4 to 64 ) in 5-21 fractions, while 8 lesions were treated with SBRT with a median BED of 62.9 Gy (range, 51.4 to 130.3) in 1-5 fractions. One month after treatment, changes in the pain score were estimated by patients. Sixteen (66.7\%) patients with 18 (64.3\%) spine lesions reported improved pain score after treatment.

\section{Local progression-free rate and overall survival}

The median follow-up duration was 11 months (range, 2 to 58 months). Local progression of the treated spine occurred in 10 lesions (35.7\%) and the median time to recurrence was 5.8 months (range, 1.1 to 25.2 months). The 1-year LPFR and OS were $67.0 \%$ and $60.7 \%$, respectively (Fig. 1). According to the use of targeted molecular therapy, 1-year LPFR was 90.0\% in patients with concurrent targeted molecular therapy and $51.3 \%$ in those without ( $p=0.019$, Fig. 2). While, the 1 -year OS for concurrent group and nonconcurrent group were $46.2 \%$ and $72.2 \%$, respectively $(p=0.114)$.

The factors associated with LPFR are described in Table 2. On univariate analysis, several factors appeared to be related to LPFR including concurrent use of target agents, cord compression, and pain response after treatment. SINS, prior irradiation, total RT dose, and dose scheme (non-SBRT vs.
Table 3. Patterns of first failure

\begin{tabular}{lcc}
\hline \multicolumn{1}{c}{ Pattern of failure } & Nonconcurrent/none & Concurrent \\
\hline Local progression & $2(13.3)$ & $0(0)$ \\
Systemic progression & $11(73.3)$ & $9(69.3)$ \\
$\begin{array}{l}\text { Both local and systemic } \\
\text { progression }\end{array}$ & $2(13.3)$ & $0(0)$ \\
No progression & $0(0)$ & $4(30.7)$ \\
\hline
\end{tabular}

Values are presented as number (\%).

SBRT) were not found to significantly influence LPFR.

On multivariate analysis, favorable pain response after treatment remained a significant factor associated with LPFR (HR, 0.19; 95\% Cl, 0.04 to 0.92), while concurrent use of targeted molecular therapy showed a tendency for improved LPFR (HR, 0.13; 95\% Cl, 0.01 to 1.16). However, presence of spinal cord compression was not an independently significant predictor.

\section{Patterns of progression}

The patterns of first failure are shown in Table 3. Local recurrence as the first site of failure was noted in four lesions with $(n=2)$ or without $(n=2)$ synchronous systemic progression. None of the lesions treated concurrently with targeted molecular therapy recurred primarily at the local site. However, the rate of systemic progression was similar between the two groups.

\section{Toxicity}

None of the patients developed grade $\geq 2$ toxicity during RT. Only grade 1 toxicities were observed in 4 patients (16.6\%). Two patients (8.3\%) reported grade 1 nausea at the time of treatment, and another 2 patients (8.3\%) complained of fatigue after treatment. 
A

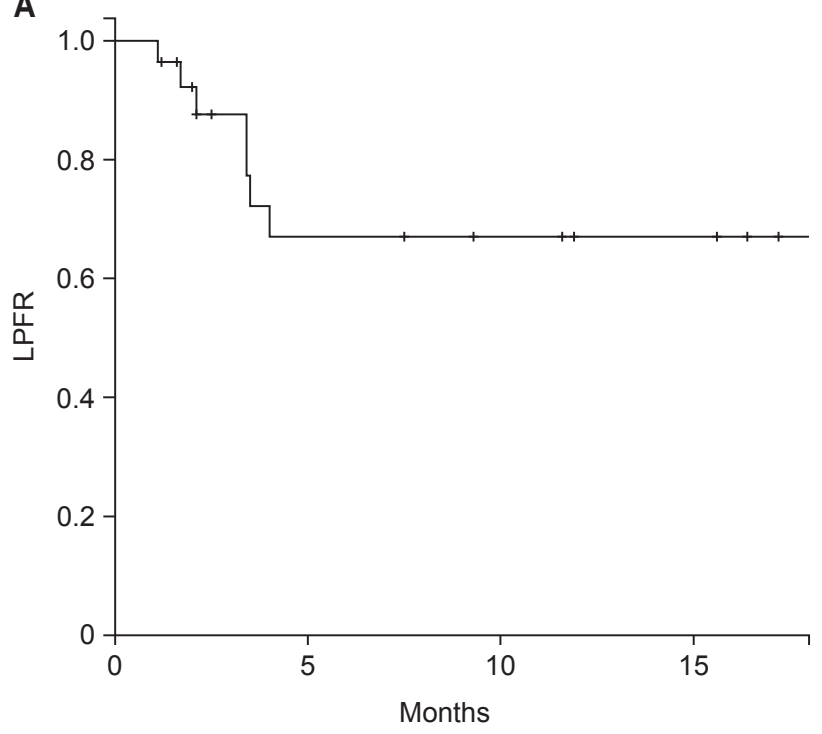

B

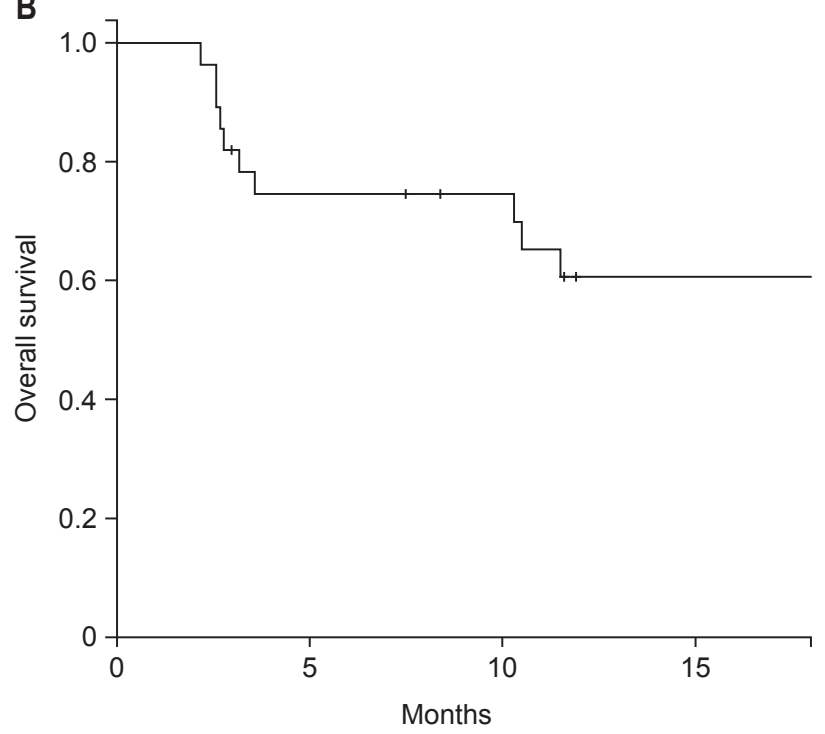

Fig. 1. (A) Local progression-free rate (LPFR) and (B) overall survival of the entire cohort.

\section{Discussion and Conclusion}

We evaluated the outcomes of spinal RT and investigated the effect of concurrent use of targeted molecular therapy for spinal metastasis from RCC. Although several studies have reported outcomes of RT for spinal metastasis from RCC, none have focused on the effect of concurrent use of targeted molecular therapy. Since distinguishing post-RT change and tumor progression is challenging, we adopted the latest recommendation by the SPINO group [14].

The 1-year LPFR was significantly improved in patients who administered concurrent targeted molecular therapy compared to patients who underwent RT only. Whereas, the 1-year OS did not differ between two groups. After adjusting for other clinical factors, concurrent use of targeted molecular therapy tended to show improved local control. The biologic background of the possibility of a synergistic effect from combination of targeted molecular therapy and RT has been previously suggested [10]. Sunitinib, the most-studied TKI in the treatment of RCC, has already been observed to potentiate the radiation-induced response. It affects T-cell priming and increases radiation sensitivity by normalizing the tumor microenvironment such as tumor vasculature. In addition, it also reduces the level of myeloid-derived suppressor cells (MDSCs) and regulatory T cells (Tregs) and co-inhibitory molecules. Although sorafenib has some immune-suppressive effects, it reduces Treg and decreases natural killer (NK) cell inhibition, resulting in immune-stimulatory effects. Pazopanib

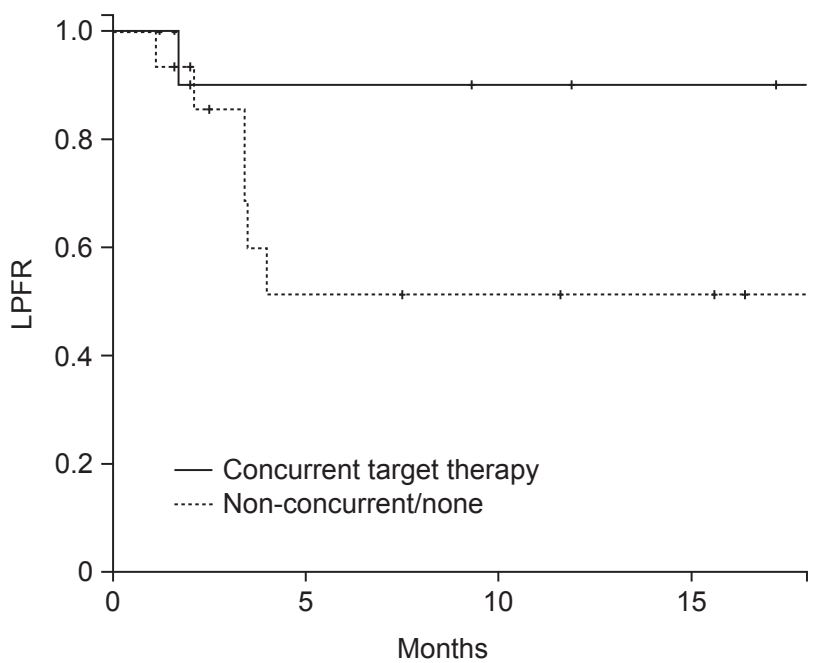

Fig. 2. Local progression-free rate (LPFR) of the patients who received concurrent targeted therapy and those who did not.

is a newer TKI, and not much is known about its mechanism. However, it is suspected that pazopanib in combination with RT might have similar effects to those of sunitinib. The mTOR inhibitors, such as temsirolimus and everolimus, are known to enhance CD8+ T cell activation. When used in combination with radiation, mTOR inhibitors might further boost the immuno-stimulatory effect.

Although the dose response relationship of radiation is well established in RCC [16], we did not observe any dose response relationship in our study. In a previous study where 24 painful 
bone metastatic lesions from RCC were treated with SBRT, applying BED ( $\alpha / \beta$ of 7$)>85$ Gy achieved faster and more durable pain relief compared to those treated with BED $<85$ Gy [17]. A more recent study comparing local control after SBRT or non-SBRT for bone metastasis from RCC reported that BED ( $\alpha / \beta$ of 7$) \geq 80$ Gy was a meaningful predictive factor [18]. The discrepancy between previous results and ours may be from the suboptimal RT dose applied in our study, for which the median BED ( $\alpha / \beta$ of 7) was 51.4 Gy (range, 31.4 to 130.3 Gy). This lower median BED was mainly due to the large portion of patients who received non-SBRT. Despite the lower dose, those who received concurrent targeted molecular therapy with RT showed a LPFR of 90\%, which is in the same order of magnitude as LPFR of previous studies using SBRT [5] and is better than LPFR from studies using non-SBRT [19]. More investigations are needed to evaluate the RT dose response relationship in the setting of concurrent use of targeted molecular therapy.

Pain response after RT was the only significant predictor of LPFR after multivariate analysis. The relationship between pain relief and local tumor control was suggested in a previous study [20]. Though not clearly disclosed, several origins of the pain from bone metastasis have been suspected [21]. The two main mechanisms, mechanical factor and chemical mediator resulting from metastatic tumor cells, are closely related to tumor burden in the metastatic site. Therefore, patients with early pain response may exhibit a more prominent decrease in tumor burden, which may be related to better local control than those without remarkable pain relief. Although this should be confirmed in a larger cohort, pain relief after RT may serve as an early predictor for local tumor control in spine metastasis from RCC.

One of the concerns regarding the use of targeted molecular therapy concurrently with radiation is the possibility of increased toxicity. In a phase II trial that tested the efficacy and toxicity of combination of sunitinib and hypofractionated RT, 28\% of patients experienced grade $\geq 3$ acute toxicities [12]. In contrast, we only observed 4 patients with grade 1 toxicities. Several factors might account for the lower toxicity rates observed in our study. Although our study only treated bone metastasis, the previous prospective trial treated lesions other than bone metastasis, such as lung and lymph nodes. Therefore, more severe toxicities may have been shown due to the critical organs near the treated lesion. In addition, the retrospective nature could have underestimated the actual incidence of toxicities. We surmise that concurrent targeted molecular therapy with radiation may be delivered safely in patients with spinal metastasis from RCC.

Several limitations should be taken into account due to the retrospective nature of our study. The radiation dose, target volume, and targeted agents were heterogeneous. In addition, the study population was small, which might make it difficult to achieve statistically significant results. Also, we divided patients into two groups according to the use of concurrent targeted molecular therapy without considering different radiation response mechanisms proven in other studies due to the small study cohort. Furthermore, the follow-up period was short and follow-up visits were irregular in some patients.

In conclusion, our results suggest the possibility of benefit from the concurrent use of targeted molecular therapy during radiation in treating spinal metastasis from RCC. However, we used this study to generate hypotheses that can be addressed by future prospective studies with larger populations.

\section{Conflict of Interest}

No potential conflict of interest relevant to this article was reported.

\section{References}

1. Levy DA, Slaton JW, Swanson DA, Dinney CP. Stage specific guidelines for surveillance after radical nephrectomy for local renal cell carcinoma. J Urol 1998;159:1163-7.

2. Uchida K, Miyao N, Masumori N, et al. Recurrence of renal cell carcinoma more than 5 years after nephrectomy. Int J Urol 2002;9:19-23.

3. Juusela H, Malmio K, Alfthan 0, Oravisto KJ. Preoperative irradiation in the treatment of renal adenocarcinoma. Scand $J$ Urol Nephrol 1977;11:277-81.

4. van der Werf-Messing B. Proceedings: carcinoma of the kidney. Cancer 1973;32:1056-61.

5. Nguyen $Q N$, Shiu AS, Rhines LD, et al. Management of spinal metastases from renal cell carcinoma using stereotactic body radiotherapy. Int J Radiat Oncol Biol Phys 2010;76:1185-92.

6. Balagamwala EH, Angelov L, Koyfman SA, et al. Single-fraction stereotactic body radiotherapy for spinal metastases from renal cell carcinoma. J Neurosurg Spine 2012;17:556-64.

7. Ghia AJ, Chang EL, Bishop AJ, et al. Single-fraction versus multifraction spinal stereotactic radiosurgery for spinal metastases from renal cell carcinoma: secondary analysis of Phase I/II trials. J Neurosurg Spine 2016;24:829-36.

8. Motzer RJ, Bander NH, Nanus DM. Renal-cell carcinoma. N Engl J Med 1996;335:865-75.

9. Mattei J, da Silva RD, Sehrt D, Molina WR, Kim FJ. Targeted therapy in metastatic renal carcinoma. Cancer Lett 
2014;343:156-60.

10. De Wolf K, Vermaelen K, De Meerleer G, Lambrecht BN, Ost P. The potential of radiotherapy to enhance the efficacy of renal cell carcinoma therapy. Oncoimmunology 2015;4:e1042198.

11. Kao J, Packer S, Vu HL, et al. Phase 1 study of concurrent sunitinib and image-guided radiotherapy followed by maintenance sunitinib for patients with oligometastases: acute toxicity and preliminary response. Cancer 2009;115:3571-80.

12. Tong CC, Ko EC, Sung MW, et al. Phase II trial of concurrent sunitinib and image-guided radiotherapy for oligometastases. PLoS One 2012;7:e36979.

13. Wilson D, Hiller L, Gray L, Grainger M, Stirling A, James N. The effect of biological effective dose on time to symptom progression in metastatic renal cell carcinoma. Clin Oncol ( $R$ Coll Radiol) 2003;15:400-7.

14. Thibault I, Chang EL, Sheehan J, et al. Response assessment after stereotactic body radiotherapy for spinal metastasis: a report from the SPIne response assessment in NeuroOncology (SPINO) group. Lancet Oncol 2015;16:e595-603.

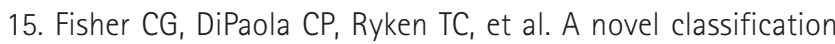
system for spinal instability in neoplastic disease: an evidencebased approach and expert consensus from the Spine Oncology Study Group. Spine (Phila Pa 1976) 2010;35:E1221-9.
16. DiBiase SJ, Valicenti RK, Schultz D, Xie Y, Gomella LG, Corn BW. Palliative irradiation for focally symptomatic metastatic renal cell carcinoma: support for dose escalation based on a biological model. J Urol 1997;158(3 Pt 1):746-9.

17. Jhaveri PM, Teh BS, Paulino AC, et al. A dose-response relationship for time to bone pain resolution after stereotactic body radiotherapy (SBRT) for renal cell carcinoma (RCC) bony metastases. Acta Oncol 2012;51:584-8.

18. Amini A, Altoos B, Bourlon MT, et al. Local control rates of metastatic renal cell carcinoma (RCC) to the bone using stereotactic body radiation therapy: is RCC truly radioresistant? Pract Radiat Oncol 2015;5:e589-96.

19. Sohn S, Chung CK, Sohn MJ, et al. Stereotactic radiosurgery compared with external radiation therapy as a primary treatment in spine metastasis from renal cell carcinoma: a multicenter, matched-pair study. J Neurooncol 2014;119:121-8.

20. Geels P, Eisenhauer E, Bezjak A, Zee B, Day A. Palliative effect of chemotherapy: objective tumor response is associated with symptom improvement in patients with metastatic breast cancer. J Clin Oncol 2000;18:2395-405.

21. Vakaet $L A$, Boterberg T. Pain control by ionizing radiation of bone metastasis. Int J Dev Biol 2004;48:599-606. 